\title{
Childhood tuberculosis treatment remains imprecise science
}

Last year, the World Health Organization released updated procedures on how best to tackle the global scourge of tuberculosis. The fourth edition of the "Treatment of tuberculosis: Guidelines" recommended, among other changes, increasing the dosage of tuberculosis medication required to treat children. But, in a sense, the new guidance provided a destination without a map: it failed to address the larger problem of how to improve the accuracy of pediatric dosing.

In recent months, researchers have pointed to a host of problems plaguing the diagnosis and treatment of tuberculosis in children, especially those younger than age 5. For example, at a June workshop held by a taskforce of the US Centers for Disease Control and Prevention, Steve Graham of the Royal Children's Hospital in Melbourne, Australia called for new and better means of pediatric tuberculosis diagnosis, which can be complicated by concurrent ailments such as malnourishment, HIV infection and pneumonia. And, in September, scientists noted that a negative result from the new interferon-gamma release assays cannot definitively rule out tuberculosis in children (Pediatr. Infect. Dis. J. 30, 817-818, 2011). Also in September, another group urged that animal models for tuberculosis "must be designed and utilized in a manner that is also pertinent to the pediatric population" by addressing age-related variance in drug metabolism (Pharmacol Res. 64, 176-179, 2011).

If pediatric tuberculosis is overlooked, it may in part have to do with the thought that children with the illness are less infectious than affected adults, so disease control programs tend to focus on older populations capable of spreading the pathogen within their communities. But, in certain regions of the world with high rates of the disease, "the average risk of [childhood infection] is about $20 \%$ per year of life in the first five years, so this is not a minor component that can be easily overlooked," says Tobias Kollmann, a pediatric infectious disease specialist at the University of British Columbia in Vancouver, Canada. "It's a huge growing problem in areas where tuberculosis is resurgent."

Current tuberculosis medications are comprised of a single, fixed-dose combination tablet that combines a number of drugs. As Graham cautioned in a paper published in March, the ratios of those medications should also vary according to a child's age and size, an issue that is also not addressed in the new guidelines (Paediatr. Respir. Rev. 12, 22-26, 2011). Currently, to treat small children the adult tablets are cut or crushed, which often leads to inaccurate dosing and inappropriate ratios.

To avoid these issues, experts say weightadjusted tablets or a liquid formulation need to be developed specifically for children, so that treatments can be more easily tailored. But, despite the fact that an estimated 1 million children currently suffer from tuberculosis, pharmaceutical companies do not see huge profit margins in addressing the problem and have not made significant investments in this area. Graham suggests that a solution is to give drug developers "the assurance that they would have a market.”

In addition, clinical trials for children need to be implemented so that child-specific formulations can move forward. "Companies are doing the studies in the adults and forgetting about the kids, and meanwhile all these children die," says Grace Aldrovandi, a pediatric infectious disease researcher at the University of Southern California-Los Angeles.

A handful of developers are bucking the trend. The German drug giant Bayer is currently testing its antibacterial drug Avelox (moxifloxacin) in a 2,400-adult phase 3 clinical trial for tuberculosis that could potentially be suitable for children. If the drug proves effective for grownups, it may be in safety trials in children by 2014, says Elizabeth Gardiner, head of market access for the New Yorkbased TB Alliance, which is cosponsoring the international study. The US pharmaceutical company Janssen Therapeutics in is in the early stages of developing a child-specific tuberculosis treatment called TMC207 and is in discussions with The IMPAACT Network, a clinical trials group, to begin trials with children.

"We're on the verge of real change when it comes to pediatric tuberculosis," Gardiner says, "and we hope that it really happens."

Julie Manoharan

\section{NIH student training overhauled after HHMI pulls funding}

As an undergraduate student at the University of Wisconsin-Madison, Amanda Herzog worked in a number of different research laboratories, where she studied everything from cartilage growth to pediatric tonsillectomy techniques to fruit fly behavior. So, when she started medical school at UW's School of Medicine and Public Health in 2007, she figured she'd keep up with her research activities. Yet, other than a three-month stint assisting oncologist Mark Albertini in his melanoma lab for a summer research program between her first and second years, Herzog has struggled to fit research into her schedule. "I had to devote my time to my studies," she says.

Herzog's passion for lab work inspired her to apply for a unique research program funded jointly by the US National
Institutes of Health (NIH) and the Howard Hughes Medical Institute (HHMI). She succeeded and became one of the 42 students chosen from hundreds of applicants for the program's 2010-2011 academic year. As an HHMI-NIH Research Scholar, she was able to defer her third year of medical school and instead study an experimental head and neck cancer drug in cellular and animal models at the $\mathrm{NIH}$ in Bethesda, Maryland.

For Herzog, the experience was a first step to fulfilling her budding career ambitions. "I would love to have my own lab down the road," she says, "and this program will help me get my foot in the door." But other aspiring medical researchers might not be so lucky. On 30 August, the NIH announced plans to replace its existing medical training programs with a new education initiative that, at least initially, will only offer around half the number of spots that were previously available.

$\mathrm{HHMI}$ has funded the Research Scholars Program - also known as the Cloister Program because the participants are housed in an old convent on the NIH campus-since its inception in 1985. But the goal all along had been simply to help get the program off the ground, notes William Galey, director of graduate science education programs for the Chevy Chase, Maryland-based nonprofit. A quarter-century later, with that mission accomplished, HHMI decided to pull its support and invest in other training efforts, such as its international student research fellowships, offered for the first time this year. "It was really time to hand the keys over to the NIH," Galey says. 